\title{
Comparisons of Condylar Movements with the Functional Occlusal Clutch and Tray Clutch Recording Methods in CADIAX ${ }^{\circledR}$ system
}

\author{
Bi-jie Han ${ }^{1}$, Hong Kang ${ }^{2}$, Lai-kui Liu ${ }^{3}$, Xin-zhu Yi ${ }^{1}$, Xiao-qing Li ${ }^{1 *}$ \\ ${ }^{1}$ Department of Oral Anatomy and Physiology, West China College of Stomatology, Sichuan University, Chengdu, China \\ ${ }^{2}$ Institute of Stomatology, Lanzhou University, Lanzhou, China \\ ${ }^{3}$ College of Stomatology, Nanjing Medical Science University, Nanjing, China
}

\begin{abstract}
Aim The purpose of this study is to compare the effects of the two clutches on recording the condylar movement.

Methodology Ten subjects (6 women, 4 men; mean age 25.4 years) participated in the study. The mandibular movement, sagittal condylar inclination angle, and transversal condylar inclination angle of each subject were recorded with the CADIAX ${ }^{\circledR}$ using the two clutches, respectively. The characteristics of the tracings of the protrusion, opening, and mediotrusion were analyzed with the $t$-test statistics at $\alpha=0.05$ level. The Kappa values were calculated for an assessment of the congruence of the tracings.
\end{abstract}

Results The results showed that the contour, direction, and dimension of the tracings in the two clutches were approximately same, but the tracings determined by the functional occlusal clutch were more regular and congruent. In the group segment recorded with the tray clutch, opening/ closing paths of one subject showed crossed and time curves of three subjects appeared peak-like changes of velocity, but none were statistically different $(P>0.05)$.

Conclusion The research suggests that the functional occlusal clutch should be preferred in the evaluation of the mandibular function, as the tracings with the tray clutch are more likely to produce false positive results.

Keywords CADIAX, temporomandibular joint, mandibular movement, functional clutch, occlusion

Received Dec. 25, 2009; Revision accepted Sep. 10, 2010

\section{Introduction}

The chief aim of recording mandibular movements is to study the functional status of the mandibular movement guided by the condyle (Okeson, 2003). The movement of the condyle is part of the whole mandibular movement. By recording the path of the condylar movement, the mandibular movement research becomes more detailed and accurate. This is meaningful for studying the physiology and pathophysiology of the mandibular functional movement in the stomatognathic system.

The Computer Aided Diagnosis Axiograph System (CADIAX ${ }^{\circledR}$, GAMMA CO, and Austria) is currently one of the most popular used instruments to study the condylar movement (Okeson,
2003; Piehslinger et al., 1991). Two kinds of clutches are often used in the CADIAX ${ }^{\circledR}$ system as the fixing components in the oral cavity to connect the system with mandible as a whole. These clutches are respectively called the tray clutch and functional occlusal clutch. The tray clutch is more often used in clinical practice because of its convenience for installing the CADIAX ${ }^{\circledR}$ recording system. However, use of the tray clutch might modify the patient's occlusal state during recording condylar movement in that it increases the occlusal vertical dimension and overlays all occlusal surface of maxillary teeth or mandibular teeth (Piehslinger et al., 1991). It has not yet been clarified which is more important between the occlusal vertical dimension and overlay in studying mandibular movement function and practice 
in clinical diagnosis. The purpose of this study was to compare the effects of the functional occlusal clutch and tray clutch on recordings of the condylar movement, and to provide guidance for clinical practice. Additionally, the correlation between occlusion and the path of the condylar movement was also explored and evaluated.

\section{Materials and Methods}

\section{Selection of Subjects}

The test subjects were selected in accordance with the following inclusion criteria:

(1) No deformities such as retruded jaw, protruded jaw, and asymmetric patterns in the maxillofacial region;

(2) Permanent dentition aligned normally and completely (except the third molar) with a normal overjet and overbite relation;

(3) No typical dental abrasion, large restorations, and prostheses such as a crown and an inlay on the occlusal surfaces;

(4) Harmonious relationship between the retruded contact position and intercuspal position without obvious occlusal interferences;

(5) No obvious oral parafunction habits such as unilateral mastication, clenching, and grinding;

(6) No temporomandibular joint (TMJ) symptoms or signs, or TMJ disorder history and no orthodontic treatment history;

According to the mentioned criteria, 10 subjects (six women and four men with a mean age of 25.4 years) were selected to participate the study.

\section{Recording Methods}

Fabrication of each of the functional clutches was made according to the following procedures. The maxillary and mandibular casts of each subject were obtained and covered with a separating medium. The functional clutch was fabricated by coating a layer of auto-polymerizing acrylic resin with a $2 \mathrm{~mm}$ thickness $(1 \mathrm{~mm}$ thick near the middle line) on the labial and buccal surfaces of the lower teeth. The upper cast was then closed to bite the lower casts, and any excess material was removed. After it hardened, the clutch was placed into the mouth, making sure that there is no interference to the occlusional movement. The handle (made of aluminum alloy) was attached to the clutch with an acrylic resin on the basis of its orientation, consistent with the median-sagittal and parallel to the occlusal plane (Figure 1).

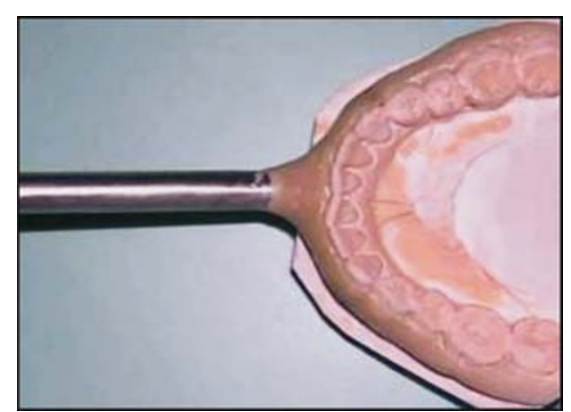

Figure 1 The self-made functional occlusal clutch

Each subject was comfortably seated in a dental chair. The recording process was carried out in the following order:

(1) Fixing the tray clutch onto the lower jaw teeth; The tray clutch filled with the silicone impression material and adhesive was seated over the lower teeth.

(2) Mounting the anatomical facebow;

(3) Mounting the mandible writing bow and adjusting the writing tips to the statistical hingeaxis; The operator guided the subject's lower jaw to the centric relation (CR) and located the reference point.

(4) Attaching the electronic flags and styli;

(5) Recording TMJ movement; The subject was guided to finish the four separate mandibular movements - protrusion, opening, right mediotrusion and left mediotrusion. The upper teeth were always contacted with the tray clutch during each movement and the starting position of each movement was at the CR. Each subject was instructed to repeat three times, with the best curve recorded.

Then, the tray clutch was removed and the functional occlusal clutch was luted to the mandibular teeth with glass ionomer cement. The reference position was relocated and the same recording procedures were repeated as described in the tray clutch method.

\section{Recordings}

The form of protrusion, opening/closing move- 
ments, and mediotrusion tracings, as well as the orientation and border of the Bennett movement, were analyzed and then compared between the two clutches.

According to the method suggested by Bernhardt et al. (2003), the characteristics of the tracings, including the tracing lengths $(<8 \mathrm{~mm}$, 8-12 $\mathrm{mm}$, or $>12 \mathrm{~mm}$ ), the degrees of the overlap of the laterotrusive and mediotrusive tracings (congruent or incongruent tracing quality), and the types of the tracings (normal, inverse, or irregular) were identified. The horizontal projections in the protrusion and opening tracings (congruent/ incongruent) were evaluated. The time and velocity curves of the two clutches were analyzed and compared.

Sagittal condyle inclination (SCI) and transversal condyle inclination (TCI) was calculated with CADIAX $^{\circledR}$ Compact under the articulator programming function mode of Artex ${ }^{\circledR}$ A-Serie. $\mathrm{SCI}$ is the projection angle between the condylar path on the sagittal and FH planes. TCI is the projection angle between the condylar path on the horizontal and sagittal planes. SCI and TCI of the two clutches were compared quantitatively.

\section{Evaluation}

The paired-samples, $t$-tests, were used to compare the condylar inclination angles determined

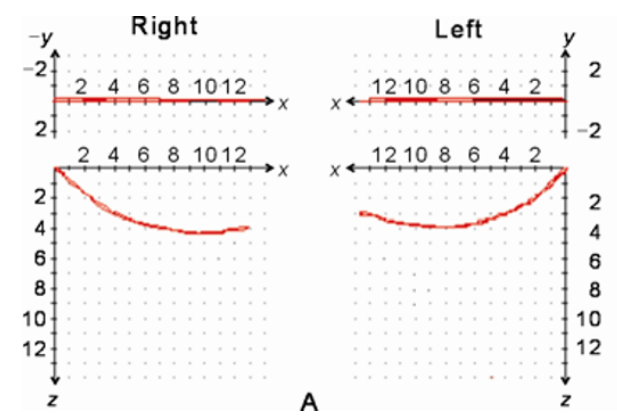

by the tray clutch and functional occlusal clutch. The Kappa values on the quantity, quality, characteristics and horizontal features in protrusion, opening/closing movements, and mediotrusion recordings were calculated to test the consistency and reliability of the mandibular tracings with two clutches. All statistical calculations were performed with SPSS 12.0 for Windows software at $\alpha=0.05$ level.

\section{Results}

We found that the self-made functional occlusion clutch could bear the weight of the lower facebow and remain stable during the recording of the mandibular movements. Though the contour of the condylar tracings of the tray clutch and functional occlusal clutch, the direction and dimension, were approximately the same, the tracings determined by the functional occlusal clutch were more regular and had a better congruence than that of the tray clutch in the 10 subjects (Figures 2A, 2B). In the tracings of one subject recorded with the tray clutch, we found a crossed change in the protrusive path on the sagittal plane (Figure 3B arrows indicated, the same to the following figures).

The Kappa values from the functional occlusal clutch and tray clutch measured with the CADIAX ${ }^{\circledR}$ system were calculated and shown in Table 1.

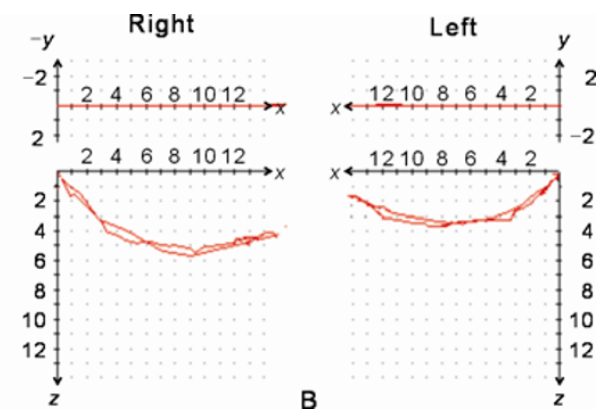

Figure 2 The similar contour, direction and dimension of the opening-closing tracings recorded with the two clutches

(A): The tracing recorded with the functional occlusal clutch; (B): The tracing recorded with the tray clutch

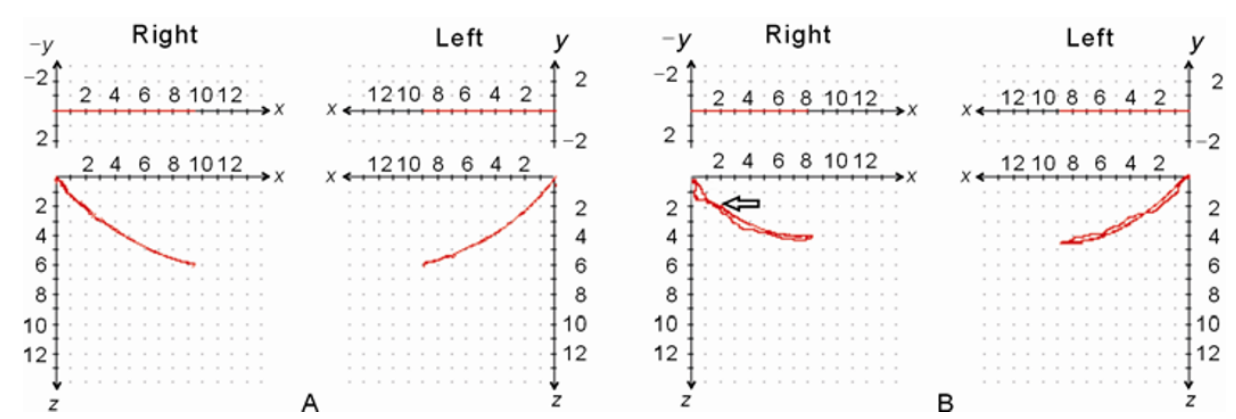

Figure 3 The protrusion tracings crossed on the sagittal plane of the tray clutch (indicated by the arrow)

(A): The tracing recorded with the functional occlusal clutch; (B): The tracing recorded with the tray clutch 
Table 1 The analysis on the congruence of the tracings recorded with the functional occlusal clutch and the tray clutch (Kappa value)

\begin{tabular}{lcccc}
\hline \multicolumn{1}{c}{ Tracing } & Lengths & Congruence & Characteristics & Horizontal \\
\hline Protrusion & 0.78 & 0.40 & 0.41 & 0.74 \\
Opening/closing & 0.60 & 0.60 & 0.41 & 0.74 \\
Mediotrusion & 0.40 & 0.52 & 0.41 & 0.55 \\
\hline
\end{tabular}

The time curves obtained with the functional occlusal clutch were smooth and stable, but three subjects' time curves recorded with the tray clutch showed peak changes of velocity at certain time points. The velocity of the tray clutch's curve increased more rapidly at 1.95 seconds than that in the recordings with functional occlusal clutch (Figure 4B). The time curves for the protrusion movement and mediotrusion showed similar results when compared with the recordings obtained in the opening/closing movements with the tray clutch. The peak time on the time curves for protrusion appeared at 1.85 seconds (Figure 5B), while on the time curves for the
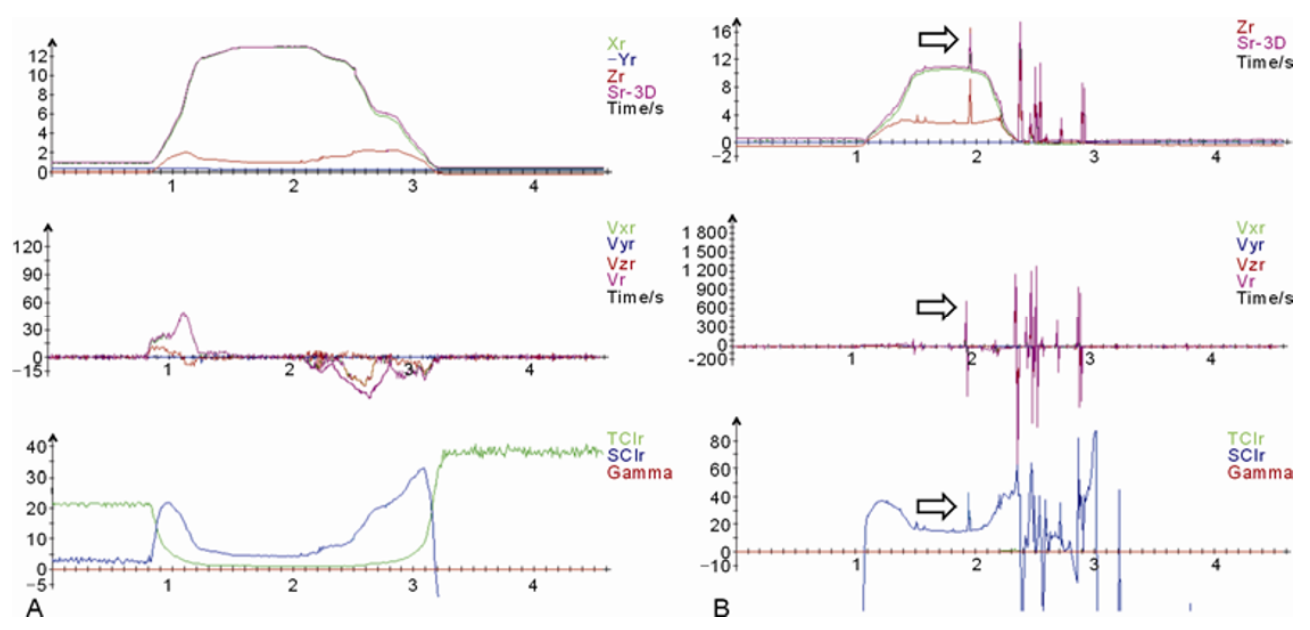

Figure 4 The time curves for the opening-closing movement

The curve obtained with the functional clutch was normal but the velocity of the curve obtained with the tray clutch rapidly increased in velocity at 1.95 seconds (indicated by the arrows). (A): The time curve recorded with the functional occlusal clutch; (B): The time curve recorded with the tray clutch.
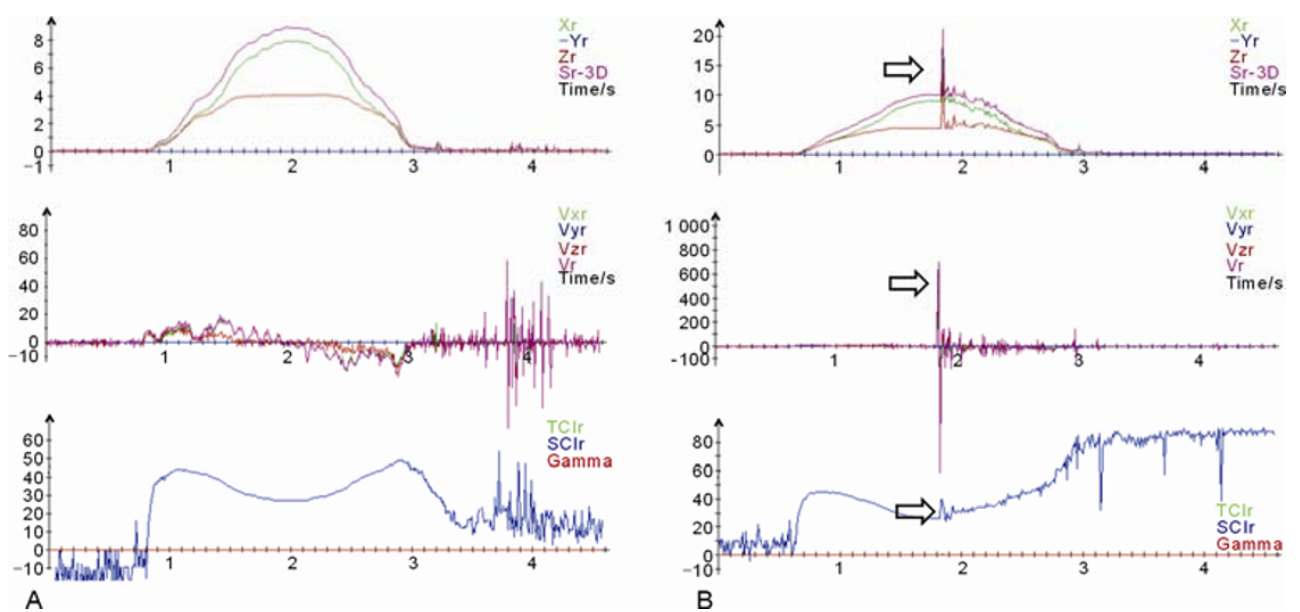

Figure 5 The time curves for the protrusion movements

The curve obtained with the functional occlusion clutch was normal but the curve obtained with the tray clutch rapidly increased in velocity at 1.85 seconds (indicated by the arrows). (A): The time curve recorded with the functional occlusal clutch; (B): The time curve recorded with the tray clutch. 

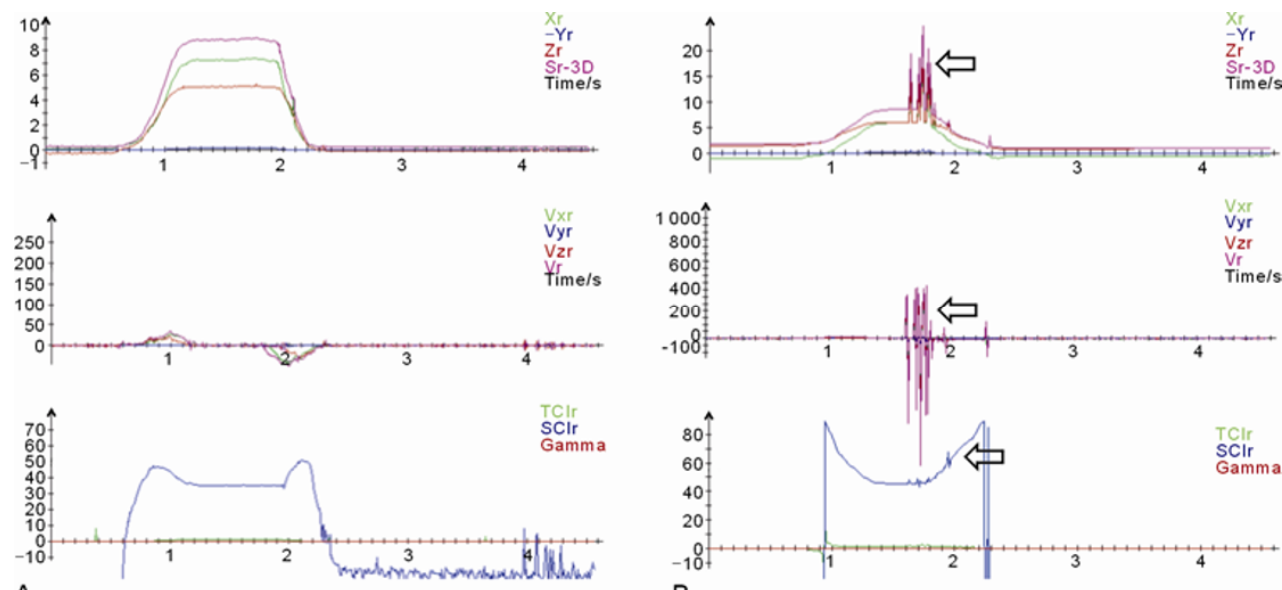

A

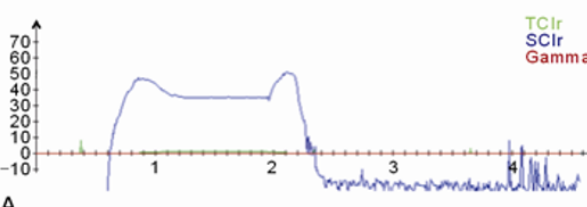

B

Figure 6 The time curves for the mediotrusion right, the curve obtained with the functional occlusal clutch was normal but the curve obtained with the tray clutch rapidly increased in velocity at 1.7 to 1.9 seconds (indicated by the arrows)

(A): The time curve recorded with the functional occlusal clutch; (B): The time curve recorded with the tray clutch.

Table 2 The comparisons of SCI and TCI ( ${ }^{\circ}$, degree) for protrusion, opening-closing, mediotrusion movements recorded with the functional occlusal clutch and the tray clutch methods

\begin{tabular}{|c|c|c|c|c|c|c|c|c|c|c|c|c|c|}
\hline \multirow[b]{2}{*}{$D$} & & \multicolumn{3}{|c|}{ Protrusion } & \multicolumn{3}{|c|}{ Opening/closing } & \multicolumn{3}{|c|}{ Mediotrusion (SCl) } & \multicolumn{3}{|c|}{ Mediotrusion (TCl) } \\
\hline & & $\bar{x} \pm s$ & $t$ & $P$ & $\bar{x} \pm s$ & $t$ & $P$ & $\bar{x} \pm s$ & $t$ & $P$ & $\bar{x} \pm s$ & $t$ & $P$ \\
\hline 1 & A & $\begin{array}{l}39.49 \pm 9.81 \\
38.17 \pm 9.04\end{array}$ & 1.58 & 0.13 & $\begin{array}{l}42.81 \pm 10.98 \\
41.80 \pm 10.95\end{array}$ & 1.91 & 0.071 & $\begin{array}{l}46.99 \pm 9.71 \\
45.35 \pm 8.78\end{array}$ & 1.70 & 0.10 & $\begin{array}{l}4.91 \pm 3.14 \\
4.69 \pm 2.92\end{array}$ & 1.71 & 0.10 \\
\hline 2 & A & $\begin{array}{l}39.92 \pm 8.93 \\
38.64 \pm 7.29\end{array}$ & 1.40 & 0.18 & $\begin{array}{l}42.33 \pm 9.50 \\
41.13 \pm 8.33\end{array}$ & 1.66 & 0.11 & $\begin{array}{l}46.39 \pm 7.64 \\
45.15 \pm 7.35\end{array}$ & 1.57 & 0.13 & $\begin{array}{l}5.74 \pm 3.09 \\
5.63 \pm 2.91\end{array}$ & 0.89 & 0.39 \\
\hline 3 & A & $\begin{array}{l}40.21 \pm 8.06 \\
38.72 \pm 6.34\end{array}$ & 1.47 & 0.16 & $\begin{array}{l}40.52 \pm 8.73 \\
39.51 \pm 7.51\end{array}$ & 1.38 & 0.18 & $\begin{array}{l}45.31 \pm 6.49 \\
44.15 \pm 6.02\end{array}$ & 1.89 & 0.07 & $\begin{array}{l}6.93 \pm 2.98 \\
6.79 \pm 2.91\end{array}$ & 0.82 & 0.42 \\
\hline 4 & B & $\begin{array}{l}38.27 \pm 7.69 \\
36.85 \pm 6.41\end{array}$ & 1.72 & 0.10 & $\begin{array}{l}38.06 \pm 7.97 \\
37.04 \pm 6.65\end{array}$ & 1.45 & 0.16 & $\begin{array}{l}43.42 \pm 6.40 \\
42.44 \pm 5.31\end{array}$ & 1.56 & 0.14 & $\begin{array}{l}7.59 \pm 2.67 \\
7.43 \pm 2.52\end{array}$ & 0.71 & 0.49 \\
\hline 5 & B & $\begin{array}{l}37.27 \pm 7.08 \\
35.84 \pm 6.07\end{array}$ & 1.63 & 0.12 & $\begin{array}{l}35.64 \pm 7.54 \\
34.70 \pm 6.42\end{array}$ & 1.68 & 0.11 & $\begin{array}{l}42.00 \pm 6.29 \\
41.26 \pm 6.59\end{array}$ & 1.30 & 0.21 & $\begin{array}{l}8.78 \pm 3.15 \\
8.52 \pm 3.02\end{array}$ & 1.70 & 0.10 \\
\hline 6 & $\begin{array}{l}\text { A } \\
\text { B }\end{array}$ & & & & & & & $\begin{array}{l}40.05 \pm 6.31 \\
39.13 \pm 5.92\end{array}$ & 1.40 & 0.18 & $\begin{array}{l}8.54 \pm 3.49 \\
8.41 \pm 3.36\end{array}$ & 1.43 & 0.17 \\
\hline
\end{tabular}

$D$ : Distance/ mm; A: the group of the functional occlusal clutch; B: the group of the tray clutch.

SCI: sagittal condyle inclination; TCI: transversal condyle inclination.

mediotrusion the rapid increase of velocity ceased at 1.7 to 1.9 seconds (Figure 6B).

Quantitative analyses on SCI and TCI of the two clutches showed that no significant differences existed in protrusion, opening-closing, mediotrusion right, and mediotrusion left (Table
2). There were also no significant differences for any condylar inclination angles of the various movements measured at any of the incremental distances on the curve in all ten subjects $(P>$ $0.05)$. 


\section{Discussion}

The analysis performed on the condylar tracings is helpful to understand the function of the temporomandibular joint. It is currently considered as an accessory diagnostic means for the diagnosis of stomatognathic dysfunctions and for functional evaluations after the application of orthodontics and orthopaedics (Slavicek, 1988; 1989a; 1989b).

In this study, the contour of the condylar tracings, the direction and dimension of the Bennett movements, which were recorded with the tray clutch and functional occlusal clutch, were similar. These findings suggest that the neuromuscular system might have some memory and dictate its behavior according to past experiences, even though the tray clutch changed the occlusal contact state during a short period of time. This is the reason why edentulous patients' previous masticatory movements are recorded for long periods, even though the rehabilitated occlusion of their completed denture work is different than that of their natural teeth. Tracings recorded with the functional occlusal clutch were more regular and congruence were better than that of the tray clutch, but no statistical differences of significance were found. A crossed change was found on the tracing recorded with the tray clutch in one subject (Figure 3B), as well as recorded peaks in velocity at certain time points in three subjects' time curves with the tray clutch (Figure 4B, 5B, 6B). The reason might be that when the subjects performed a mandibular eccentric movement that kept their maxillary teeth in contact with the tray clutch, they felt discomfort and increased their masticatory muscular tones through the protective reflex of the periodontal proprioceptor. The interferential waves observed in the tracings could be a false positive reaction resulting from the muscular tension induction. This was in agreement with Petrie's et al. view (Petrie et al., 2003). Another possible explanation might be that when the relatively thick layer of the polyvinyl siloxane material was used to affix the tray clutch to the teeth, this elastomeric material could exhibit a resiliency that captured any minor movement occurring between the teeth and the clutch during recordings. This might account for some of the changes found in our results obtained with the tray clutch recording.

The recording of the condyle tracings can be applied to other aspects, for example, the calculated parameter can be used to set the condylar inclination of partly or fully adjustable articulators. Whether or not the angles of the condylar path are determined by only one factor, such as occlusion, is not clear. Simonet et al. found that changing a patient's occlusion would not produce marked changes of TCI (Simonet, 1981). Alsawaf and Fischer-Brandies et al. also found that no significant variations of SCI were produced after changing the patient's occlusion (Alsawaf and Garlapo, 1992; Fischer-Brandies et al., 1992). Although no statistically significant differences, between the recordings, were found in SCI and TCI with the tray clutch and functional occlusal clutch $(P>0.05)$, specific changes were observed in the tracings with the tray clutch. The results supported the conclusion by Simonet and Alsawaf et al. These findings suggested the formation of the angle of the condylar path might be codetermined by an integration of factors, such as the anatomic structure of the TMJ, elasticity and tensity of the joint capsule and ligament, the contraction of the masticatory muscles and changes of occlusion. Thus, the effect of temporary changes of the occlusal factor on the condylar path angle will be limited. However, it is certain that a prolonged change of the occlusal factor will result in an unavoidable change of the condylar path. Koak et al, in their research on open bite malocclusion, found that these patients had a more relatively flattened condyle inclination than that of the normal subjects (Koak, 2000). Stamm et al. also found that the sagittal condylar inclination of the patients with an Angle II/2 was steeper than that of the subjects in the normal group (Stamm et al., 1998).

In this research we found that self-made functional clutch could bear the weight of the lower facebow and maintain stable during the whole mandibular recordings. Thus, the self-made functional clutch can generally be used in prosthodontic and orthodontic clinics due to its simple fabrication and low price. However, the functional occlusion clutch needs to be made for an individual patient and takes much chair-side time. 
Therefore, for a rapid diagnosis and collection of the articulator information, the tray clutch is preferable.

\section{Conclusion}

The following conclusions can be drawn from this research on the comprehensive comparisons between the condylar tracings recorded with the tray clutch and self-made functional occlusal clutch methods. No statistically significant differences exist in the condylar tracings recorded with the tray clutch and the functional occlusal clutch, but the tracings recorded with the tray clutch are more likely to produce false positive results. Thus, the functional occlusion clutch is the preferred method in the diagnosis of TMJ disorders and the evaluation of the mandibular function. Due to its simple fabrication and low cost, the self-made functional clutch can generally be used in clinics for the diagnosis and evaluation of stomatognathic dysfunctions.

\section{References}

Alsawaf MM, Garlapo DA (1992). Influence of tooth contact on the path of condylar movements. $J$ Prosthet Dent, 67(3): 394-400.

Bernhardt O, Küppers N, Rosin M, Meyer G (2003). Comparative tests of arbitrary and kinematic transverse horizontal axis recordings of mandibular movements. J Prosthet Dent, 89(2): 175-179.

Fischer-Brandies H, Kluge G, Burckhardt R, Heel R (1992). The effect of the interocclusal space on temporomandibular recordings. A study with the use of the Stereognathograph PC. Dtsch Zahn Mund Kieferheilkd Zentralbl, 80(3): 153-157.

Koak JY (2000). A study on the mandibular movement of anterior openbite patients. J Oral Rehabil, 27(9): 817-822.

Okeson JP (2003). Diagnosis of temporomandibular Disorders // JP Okeson ed. Management of Temporomandibular Disorders and Occlusion. 5th ed. St. Louis: C.V. Mosby, pp321.

Petrie C, Woolsey G, Williams K (2003). Comparison of recordings obtained with computerized axiography and mechanical pantography at 2 time intervals. $J$ Prosthodont, 12(2): 102-110.

Piehslinger E, Celar AG, Celar RM, Slavicek R (1991). Computerized axiography: principles and methods. Cranio, 9(4): 344-355.

Simonet PF (1981). Influence of TMJ dysfunction on Bennett movement as recorded by a modified pantograph. Part III: Progress report on the clinical study. J Prosthet Dent, 46(6): 652-661.

Slavicek R (1988). Clinical and instrumental functional analysis for diagnosis and treatment planning. Part 7: Computer aided axiography. J Clin Orthod, 22(12): 776-787.

Slavicek R (1989a). Clinical and instrumental functional analysis for diagnosis and treatment planning. Part 8: Case studies in CADIAX ${ }^{\circledR} . J$ Clin Orthod, 23(1): 4245.

Slavicek R (1989b). Clinical and instrumental functional analysis for diagnosis and treatment planning. Part 9: Removable splint therapy. J Clin Orthod, 23(2): 9097.

Stamm T, Vehring A, Ehmer U, Bollmann F (1998). Computer-aided axiography of asymptomatic individual with Class II/2. J Orofac Orthop, 59(4): 237-245.

\footnotetext{
*Corresponding author: Xiao-qing Li

Address: Department of Oral Anatomy and Physiology, West China College of Stomatology, Sichuan University, 14 Renmin South Road Section 3, Chengdu 610041, China

Tel: $862885501483 \quad$ Fax: $862885582167 \quad$ E-mail: xuanxqli@163.com
} 